\title{
Global Stability of the Rarefaction Wave of a One-Dimensional Model System for Compressible Viscous Gas
}

\author{
Akitaka Matsumura ${ }^{1}$ and Kenji Nishihara ${ }^{2}$ \\ 1 Department of Mathematics, Kanazawa University, Kanazawa 920, Japan \\ ${ }^{2}$ School of Political Science and Economics, Waseda University, Tokyo 169, Japan
}

Received May 3, 1991; in revised form June 4, 1991

\begin{abstract}
This paper is concerned with the asymptotic behavior toward the rarefaction wave of the solution of a one-dimensional barotropic model system for compressible viscous gas. We assume that the initial data tend to constant states at $x= \pm \infty$, respectively, and the Riemann problem for the corresponding hyperbolic system admits a weak continuous rarefaction wave. If the adiabatic constant $\gamma$ satisfies $1 \leqq \gamma \leqq 2$, then the solution is proved to tend to the rarefaction wave as $t \rightarrow \infty$ under no smallness conditions of both the difference of asymptotic values at $x= \pm \infty$ and the initial data. The proof is given by an elementary $L^{2}$-energy method.
\end{abstract}

\section{Introduction}

Subsequent to [10] and [11], we consider the Cauchy problem of a one-dimensional barotropic model system for compressible viscous gas. Our problem is described as

$$
\left\{\begin{array}{l}
v_{t}-u_{x}=0 \\
u_{t}+p(v)_{x}=\mu\left(\frac{u_{x}}{v}\right)_{x} \\
p(v)=a v^{-\gamma}, \quad x \in R, \quad t \in R_{+}=(0, \infty)
\end{array}\right.
$$

with the initial data

$$
(v, u)(0, x)=\left(v_{0}, u_{0}\right)(x),
$$

where $v(>0)$ is the specific volume, $u$ is the velocity, $\mu(>0)$ is the constant coefficient of viscosity and $p$ is the pressure given by $p=a v^{-\gamma}$ for a constant $a>0$ and the adiabatic constant $\gamma \geqq 1$. We assume the initial data asymptotically tend to the 
constant states at $x= \pm \infty$ :

$$
\lim _{x \rightarrow \pm \infty}\left(v_{0}, u_{0}\right)(x)=\left(v_{ \pm}, u_{ \pm}\right), \quad v_{ \pm}>0
$$

The asymptotic behavior as $t \rightarrow \infty$ of the solution is closely related to that of the Riemann problem for the corresponding hyperbolic conservation law:

$$
\left\{\begin{array}{l}
v_{t}-u_{x}=0 \\
u_{t}+p(v)_{x}=0
\end{array}\right.
$$

with

$$
(v, u)(0, x)=\left(v_{0}^{R}, u_{0}^{R}\right)(x) \equiv \begin{cases}\left(v_{-}, u_{-}\right) & x<0 \\ \left(v_{+}, u_{+}\right) & x>0 .\end{cases}
$$

For a state $\left(v_{-}, u_{-}\right)\left(v_{-}>0, u_{-} \in \mathbf{R}\right)$, we define in a suitable neighborhood $\omega \subset R_{v, u}^{2}$ of $\left(v_{-}, u_{-}\right)$,

and

$$
\left\{\begin{array}{l}
R_{1}\left(v_{-}, u_{-}\right)=\left\{(v, u) \in \omega ; u=u_{-}-\int_{v-}^{v} \lambda_{1}(s) d s, u \geqq u_{-}\right\} \\
R_{2}\left(v_{-}, u_{-}\right)=\left\{(v, u) \in \omega ; u=u_{-}-\int_{v-}^{v} \lambda_{2}(s) d s, u \geqq u_{-}\right\}
\end{array}\right.
$$

$$
R R\left(v_{-}, u_{-}\right)=\left\{(v, u) \in \omega ; u \geqq u_{-}-\int_{v-}^{v} \lambda_{1}(s) d s, u \geqq u_{-}-\int_{v-}^{v} \lambda_{2}(s) d s\right\},
$$

where $\lambda_{1}(v)=-\sqrt{-p^{\prime}(v)}$ and $\lambda_{2}(v)=\sqrt{-p^{\prime}(v)}$ are the distinct eigenvalues of the matrix $\left(\begin{array}{cc}0 & -1 \\ p^{\prime}(v) & 0\end{array}\right)$. It is well-known that if $\left(v_{+}, u_{+}\right) \in R R\left(v_{-}, u_{-}\right)$, then the Riemann problem (1.4) with (1.5) admits a continuous weak solution of the form $\left(v^{R}, u^{R}\right)(x / t)$ (we call it "the rarefaction wave" for simplicity), which consists of three constant states and the centered rarefaction waves connecting the constant states (see Lax [6]).

We showed in [11] that the solution $(v, u)$ of the original system (1.1)-(1.3) tends to the rarefaction wave $\left(v^{R}, u^{R}\right)(x / t)$ provided both $\left|\left(v_{+}, u_{+}\right)-\left(v_{-}, u_{-}\right)\right|$and $\left(v_{0}-v_{0}^{R}, u_{0}-u_{0}^{R}\right),\left(v_{0}, u_{0}\right)_{x} \in L^{2}$ are sufficiently small. Further in [5], we succeeded in removing the smallness condition for the initial data. Our purpose in the present paper is to show that, when $1 \leqq \gamma \leqq 2$, the solution $(v, u)$ asymptotically behaves as $\left(v^{R}, u^{R}\right)(x / t)$ without smallness conditions of both the initial data and $\mid\left(v_{+}, u_{+}\right)-$ $\left(v_{-}, u_{-}\right) \mid$. Our results are precisely as follows.

Theorem 1.1. Let $1 \leqq \gamma \leqq 2$. If $\left(v_{+}, u_{+}\right) \in R R\left(v_{-}, u_{-}\right)$and $\left(v_{0}-v_{0}^{R}, u_{0}-u_{0}^{R}\right) \in L^{2}$, $\left(v_{0 x}, u_{0 x}\right) \in L^{2}$ and $v_{0}>0$, then the Cauchy problem (1.1), (1.2) with (1.3) has a unique global solution $(v, u)$ in time satisfying

$$
\begin{aligned}
\left(v-v^{R}, u-u^{R}\right) & \in C\left([0, \infty) ; L^{2}\right) \cap L^{\infty}\left(R_{+} ; L^{2}\right), \\
(v, u)_{x} & \in C\left([0, \infty) ; L^{2}\right) \cap L^{\infty}\left(R_{+} ; L^{2}\right) \cap L^{2}\left(R_{+} \times R\right), \\
u_{x x} \in L^{2}\left(R_{+} \times R\right) &
\end{aligned}
$$


and

$$
\lim _{t \rightarrow \infty} \sup _{R}\left|(v, u)(t, x)-\left(v^{R}, u^{R}\right)(x / t)\right|=0 .
$$

The asymptotic behaviors of the solutions of the single Burgers equations were originally investigated by Il'in and Oleinik [2] (cf. $[12,1]$ ). For the system, those toward the traveling wave solutions with shock profile were studied by $[10,5,7]$. It is still open when the asymptotic state is expected to be the superposition of both shock and rarefaction waves.

Our plan of this paper is as follows. In the next section, we construct a smooth approximate solution of the Riemann problem, which is the slight refinement of that in the preceding paper [11]. In the last two sections, we reformulate our problem and establish the a priori estimates by an elementary $L^{2}$-energy method with the aid of the techniques in $[3,9]$.

\section{Smooth Approximate Solution of the Riemann Problem}

In the same situations as [11], we start with the Riemann problem for the typical Burgers equation:

$$
\left\{\begin{array}{l}
w_{t}^{R}+w^{R} w_{x}^{R}=0 \\
w^{R}(0, x)=w_{0}^{R}(x) \equiv \begin{cases}w_{-} & x<0 \\
w_{+} & x>0\end{cases}
\end{array}\right.
$$

with $w_{-}<w_{+}$. As is well-known, (2.1) has a continuous weak solution of the form $w^{R}(x / t)$ given by

$$
w^{R}(\xi)= \begin{cases}w_{-} & \xi \leqq w_{-} \\ \xi & w_{-} \leqq \xi \leqq w_{+} \\ w_{+} & \xi \geqq w_{+} .\end{cases}
$$

We approximate $w^{R}(x / t)$ by the solution of the following problem:

$$
\left\{\begin{array}{l}
w_{t}+w w_{x}=0 \\
w(0, x)=w_{0}(x) \equiv \hat{w}+\tilde{w} \cdot \kappa_{q} \int_{0}^{\varepsilon x}\left(1+y^{2}\right)^{-q} d y
\end{array}\right.
$$

where $\hat{w}=\left(w_{+}+w_{-}\right) / 2, \tilde{w}=\left(w_{+}-w_{-}\right) / 2>0, \varepsilon>0$ is a small constant to be determined later and $\kappa_{q}$ is a constant such that $\kappa_{q} \int_{0}^{\infty}\left(1+y^{2}\right)^{-q} d y=1$ for each $q>3 / 2$.
Then we have

Lemma 2.1. If $w_{-}<w_{+}$, then the problem (2.3) has a unique smooth global solution $w(t, x)$ in time satisfying the following:

$$
w_{-}<w(t, x)<w_{+}, \quad w_{x}(t, x)>0, \quad(t, x) \in R_{+} \times R .
$$

(ii) For any $p(1 \leqq p \leqq \infty)$ there exists a constant $C_{p, q}$ such that 


$$
\begin{aligned}
\left\|w_{x}(t, \cdot)\right\|_{L^{p}} & \leqq C_{p, q} \min \left(\varepsilon^{1-1 / p} \tilde{w}, \tilde{w}^{1 / p} t^{-1+1 / p}\right), \\
\left\|w_{x x}(t, \cdot)\right\|_{L^{p}} & \leqq C_{p, q} \min \left(\varepsilon^{2-1 / p} \tilde{w}, \varepsilon^{(1-1 / 2 q)(1-1 / p)} \tilde{w}^{-(p-1) / 2 p q} t^{-1-(p-1) / 2 p q}\right), \quad t \in R_{+} .
\end{aligned}
$$

(iii) If $w_{-}>0$, then for some positive constant $C_{q}$,

$$
\begin{aligned}
\left|w(t, x)-w_{-}\right| & \leqq C_{q} \tilde{w}\left(1+(\varepsilon x)^{2}\right)^{-q / 3}\left(1+\left(\varepsilon w_{-} t\right)^{2}\right)^{-q / 3} \\
\left|w_{x}(t, x)\right| & \leqq C_{q} \varepsilon \tilde{w}\left(1+(\varepsilon x)^{2}\right)^{-q / 2}\left(1+\left(\varepsilon w_{-} t\right)^{2}\right)^{-q / 2}, \quad x \leqq 0, \quad t \in R_{+} .
\end{aligned}
$$

(iv) If $w_{+}<0$, then for some positive constant $C_{q}$

$$
\begin{aligned}
\left|w(t, x)-w_{+}\right| & \leqq C_{q} \tilde{w}\left(1+(\varepsilon x)^{2}\right)^{-q / 3}\left(1+\left(\varepsilon w_{+} t\right)^{2}\right)^{-q / 3}, \\
\left|w_{x}(t, x)\right| & \leqq C_{q} \varepsilon \tilde{w}\left(1+(\varepsilon x)^{2}\right)^{-q / 2}\left(1+\left(\varepsilon w_{+} t\right)^{2}\right)^{-q / 2}, \quad x \geqq 0, \quad t \in R_{+} .
\end{aligned}
$$

(v) $\lim _{t \rightarrow \infty} \sup _{R}\left|w(t, x)-w^{R}(x / t)\right|=0$.

The proof is quite similar to Lemma 2.1 in [11], in which we took the initial data $w_{0}(x)=\hat{w}+\tilde{w} \tanh x$. Since we have taken the new one in (2.3), it holds

$$
\left|w_{0}^{\prime \prime}(x)\right| \leqq 2 q \varepsilon\left(\varepsilon \tilde{w} \kappa_{q}\right)^{-1 / 2 q} w_{0}^{\prime}(x)^{1+1 / 2 q}
$$

from which (ii) $)_{2}$ follows. Hence $\left\|w_{x x}(t, \cdot)\right\|_{L^{p}}$ is integrable on $R_{+}$for $p>1$, which is the crucial different point from the preceding.

We now approximate the weak solution $\left(v^{R}, u^{R}\right)(x / t)$ of $(1.4),(1.5)$ by using the smooth solution $w(t, x)$ of (2.3). The procedure is the same as that in [11]. If $\left(v_{+}, u_{+}\right) \in R R\left(v_{-}, u_{-}\right)$, then there is a unique $(\bar{v}, \bar{u}) \in R_{1}\left(v_{-}, u_{-}\right)$satisfying $\left(v_{+}, u_{+}\right) \in$ $R_{2}(\bar{v}, \bar{u})$ and the continuous weak solution of the Riemann problem (1.4) with (1.5) is exactly given by

where

$$
\left(v^{R}, u^{R}\right)(x / t)=\left(v_{1}^{R}+v_{2}^{R}-\bar{v}, u_{1}^{R}+u_{2}^{R}-\bar{u}\right)(x / t)
$$

$$
\begin{gathered}
\lambda_{1}\left(v_{1}^{R}(\xi)\right)=w_{1}^{R}(\xi) \quad\left(\text { respectively } \lambda_{2}\left(v_{2}^{R}(\xi)\right)=w_{2}^{R}(\xi)\right) \\
u_{1}^{R}(\xi)=u_{-}-\int_{v_{-}^{R}(\xi)}^{v_{2}^{R}(\xi)} \lambda_{1}(s) d s \quad\left(\text { respectively } u_{2}^{R}(\xi)=\bar{u}-\int_{\bar{v}}^{v^{R}} \lambda_{2}(s) d s\right)
\end{gathered}
$$

and $w_{1}^{R}(\xi)$ (respectively $w_{2}^{R}(\xi)$ ) is given by (2.2) with

$$
w_{1-}=\lambda_{1}\left(v_{-}\right), \quad w_{1+}=\lambda_{1}(\bar{v}) \quad\left(\text { respectively } w_{2-}=\lambda_{2}(\bar{v}), w_{2+}=\lambda_{2}\left(v_{+}\right)\right) .
$$

Along this process of (2.4)-(2.7), we define $(V, U)(t, x)$ by

$$
\left\{\begin{aligned}
(V, U)(t, x) & =\left(V_{1}+V_{2}-\bar{v}, U_{1}+U_{2}-\bar{u}\right)(t, x) \\
\lambda_{1}\left(V_{1}\right) & =w_{1}(t, x), \quad \lambda_{2}\left(V_{2}\right)=w_{2}(t, x) \\
U_{1} & =u_{-}-\int_{v-}^{V_{1}} \lambda_{1}(s) d s, \quad U_{2}=\bar{u}-\int_{\bar{v}}^{V_{2}} \lambda_{2}(s) d s,
\end{aligned}\right.
$$

where $w_{1}$ (respectively $w_{2}$ ) is the solution of (2.3) with (2.7). It is easily seen that both $\left(V_{1}, U_{1}\right)$ and $\left(V_{2}, U_{2}\right)$ are smooth exact solutions of $(1.4)$ and that $(V, U)$ satisfies

$$
\left\{\begin{array}{c}
V_{t}-U_{x}=0 \\
U_{t}+p(V)_{x}=g(V)_{x},
\end{array}\right.
$$


where $g(V)=p(V)-p\left(V_{1}\right)-p\left(V_{2}\right)+p(\bar{v})$. We note $g(V) \equiv 0$ if $\left(v_{+}, u_{+}\right) \in R_{1}\left(v_{-}, u_{-}\right)$ or $\left(v_{+}, u_{+}\right) \in R_{2}\left(v_{-}, u_{-}\right)$.

Let $\delta=\left|v_{+}-v_{-}\right|+\left|u_{+}-u_{-}\right|$. Then $(V, U)$ satisfies the following, due to Lemma 2.1 .

Lemma 2.2. ( $V, U)$ given by (2.8) satisfies the following:

(i) $V_{t}>0, \quad(t, x) \in R_{+} \times R$.

(ii) For some constant $C$

$$
\left|V_{x}\right| \leqq C V_{t}, \quad V_{t} \leqq C \varepsilon \delta, \quad(t, x) \in R_{+} \times R .
$$

(iii) If $\tilde{w}_{i}=\left(w_{i+}-w_{i-}\right) / 2 \neq 0(i=1,2)$, then

$$
\left\|g(V)_{x}\right\|_{L^{p}} \leqq C_{p, q} \varepsilon^{1-1 / p} \tilde{w}_{1} \tilde{w}_{2}\left\{\left(1+\left(\varepsilon w_{2-} t\right)^{2}\right)^{-q / 3}+\left(1+\left(\varepsilon w_{1+} t\right)^{2}\right)^{-q / 3}\right\}
$$

for $t \in R_{+}$and

$$
\int_{0}^{\infty}\left\|g(V)_{x}\right\|_{L^{p}} d t \leqq C_{p, q} \delta^{2} \varepsilon^{-1 / p}
$$

(iv) $\left\|V_{x}(t, \cdot)\right\|_{L^{p}},\left\|U_{x}(t, \cdot)\right\|_{L^{p}} \leqq C_{p, q} \min \left(\delta \varepsilon^{1-1 / p}, \delta^{1 / p}(1+t)^{-1+1 / p}\right)$ for $t \in R_{+}$.

(v) $\left\|V_{x x}(t, \cdot)\right\|_{L^{p}},\left\|U_{x x}(t, \cdot)\right\|_{L^{p}}$

$$
\leqq C_{p, q}\left\{\delta^{-(p-1) / 2 p q} \varepsilon^{(1-1 / 2 q)(1-1 / p)}(1+t)^{-1-(p-1) / 2 p q}+\delta^{1 / p}(1+t)^{-2+1 / p}\right\}
$$

for $t \in R_{+}$and, especially, for $p>1$,

$$
\int_{0}^{\infty}\left\|V_{x x}(t, \cdot)\right\|_{L^{p}} d t, \int_{0}^{\infty}\left\|U_{x x}(t, \cdot)\right\|_{L^{p}} d t \leqq C_{p, q} \delta^{-(p-1) / 2 p q} .
$$

(vi) $\lim _{t \rightarrow \infty} \sup _{R}\left|(V, U)(t, x)-\left(v^{R}, u^{R}\right)(x / t)\right|=0$.

\section{Reformulation of the Problem}

Making use of the approximate solution $(V, U)$ constructed in the preceding section, we rewrite the Cauchy problem (1.1) with (1.2) by the change of variables $(v, u)=(V+\varphi, U+\psi)$ as follows:

$$
\begin{gathered}
\left\{\begin{array}{l}
\varphi_{t}-\psi_{x}=0 \\
\psi_{t}+(p(V+\varphi)-p(V))_{x}-\mu\left(\frac{u_{x}}{v}-\frac{U_{x}}{V}\right)_{x}=G_{x},
\end{array}\right. \\
(\varphi, \psi)(0, x)=\left(\varphi_{0}, \psi_{0}\right)(x) \equiv\left(v_{0}(x)-V(0, x), u_{0}(x)-U(0, x)\right),
\end{gathered}
$$

where

$$
\begin{aligned}
G_{x} & =\mu\left(\frac{U_{x}}{V}\right)_{x}-g(V)_{x} \\
g(V) & =p(V)-p\left(V_{1}\right)-p\left(V_{2}\right)+p(\bar{v}) .
\end{aligned}
$$


We seek the solution of (3.1), (3.2) in the set of functions $X(0, \infty)$ where, for $0<T \leqq \infty$,

$X(0, T)=\left\{(\varphi, \psi) \in C\left([0, T) ; H^{1}\right) ; \varphi_{x} \in L^{2}\left(0, T ; L^{2}\right), \psi_{x} \in L^{2}\left(0, T ; H^{1}\right)\right.$ and $\left.0<V+\varphi<\infty\right\}$.

Here $H^{k}(k \geqq 0)$ denote the usual Sobolev space with the norm $\|\cdot\|_{k}$. In particular, $H^{0}=L^{2}$ with $\|\cdot\|_{0}=\|\cdot\|$.

For the proof of Theorem 1 it suffices to show

Proposition 3.1. Let $1 \leqq \gamma \leqq 2$. Then, there exists a unique global solution $(\varphi, \psi) \in X(0, \infty)$ and a positive constant $C_{0}$ satisfying

$$
C_{0}^{-1} \leqq v(t, x) \leqq C_{0}
$$

and

$$
\sup _{t \geqq 0}\|(\varphi, \psi)(t)\|_{1}^{2}+\int_{0}^{\infty}\left(\left\|\sqrt{V_{t}} \varphi(\tau)\right\|^{2}+\left\|\varphi_{x}(\tau)\right\|^{2}+\left\|\psi_{x}(\tau)\right\|_{1}^{2}\right) d \tau \leqq C_{0}\left\|\varphi_{0}, \psi_{0}\right\|_{1}^{2} .
$$

Proposition 3.1 is obtained by the combination of the existence and uniqueriess of the local solution in time with the continuation process to construct the global solution (cf. $[8,10,11]$ etc.). In the later section, we devote ourselves to the following a priori estimates.

Proposition 3.2. (A priori estimates) When $1 \leqq \gamma \leqq 2$, suppose the problem (3.1), (3.2) has a solution $(\varphi, \psi) \in X(0, T)$ for some $T>0$. Let $\tilde{v}=v / V$, then there exists a positive constant $C_{1}$ independent of $T$ such that

$$
\begin{gathered}
C_{1}^{-1} \leqq v(t, x) \leqq C_{1} \quad \text { for } \quad(t, x) \in[0, T] \times R, \\
\|(\tilde{v}-1, \psi)(t)\|_{1}^{2}+\int_{0}^{t}\left\|\sqrt{V_{t}}(\tilde{v}-1)(\tau)\right\|^{2}+\left\|\tilde{v}_{x}(\tau)\right\|^{2}+\left\|\psi_{x}(\tau)\right\|_{1}^{2} d \tau \\
\leqq C_{1}\left(\left\|\left(v_{0} / V(0, \cdot)-1, \psi_{0}\right)\right\|_{1}^{2}+1\right) \quad \text { for } \quad t \in[0, T] .
\end{gathered}
$$

If we note $\tilde{v}_{x}=\frac{\varphi_{x}}{V}-\frac{V_{x}}{V}(\tilde{v}-1)$, it suffices to prove Proposition 3.2 for the proof of Proposition 3.1.

\section{A Priori Estimates}

Throughout this section we suppose the problem (3.1) with (3.2) has a solution $(\varphi, \psi) \in X(0, T)$ for some $T>0$. We write $C$ as generic positive constants which may be depend on $\tilde{w}_{i}(i=1,2)$ and $\varepsilon$, but are independent of $t(0 \leqq t \leqq T)$ and $(\varphi, \psi)$. $C_{j}(a, b, \ldots), j=2,3, \ldots$, denote some positive constants depending on $a, b, \ldots$. Also, we abbreviate the integrand $R$ without confusion.

Lemma 4.1. For sufficiently small $\varepsilon>0$,

$$
\begin{aligned}
& \frac{1}{2}\|\psi(t)\|^{2}+\int \Phi(v, V)(t, x) d x+C^{-1} \int_{0}^{t} \int\left(p(V+\varphi)-p(V)-p^{\prime}(V) \varphi\right) V_{t}+\frac{\psi_{x}^{2}}{v} d x d \tau \\
& \leqq \frac{1}{2}\left\|\psi_{0}\right\|^{2}+\int \Phi\left(v_{0}(x), V(0, x)\right) d x+C \equiv C_{2}\left(\left\|\varphi_{0}\right\|,\left\|\psi_{0}\right\|\right)
\end{aligned}
$$




$$
\int_{0}^{t} \int\left|\frac{\varphi \psi_{x} V_{t}}{v V}\right| d x d \tau \leqq C_{2}\left(\left\|\varphi_{0}\right\|,\left\|\psi_{0}\right\|\right)
$$

where $\Phi(v, V)=p(V)(v-V)-\int_{V}^{v} p(\eta) d \eta \geqq 0$.

Remark 4.1. If we put $\tilde{v}=v / V$, then $\Phi(v, V)=\tilde{\Phi}(\tilde{v}) / V^{\gamma-1}$ and $p(V+\varphi)-p(V)-$ $p^{\prime}(V) \varphi=\left(\tilde{v}^{-\gamma}-1+\gamma(\tilde{v}-1)\right) / V^{\gamma}$, where

$$
\tilde{\Phi}(\eta)= \begin{cases}\eta-1-\log \eta & \gamma=1 \\ \eta-1+\left(\eta^{1-\gamma}-1\right) /(\gamma-1) & \gamma>1\end{cases}
$$

Here we put $a=1$ without loss of generality. Hence (4.1) is rewritten as

$$
\begin{aligned}
& \frac{1}{2}\|\psi(t)\|^{2}+\int V(t, x)^{1-\gamma} \tilde{\Phi}(\tilde{v}(t, x)) d x+C^{-1} \int_{0}^{t} \int \frac{V_{t}}{V^{\gamma}}\left(\frac{1}{\tilde{v}^{\gamma}}-1+\gamma(\tilde{v}-1)\right)+\frac{\psi_{x}^{2}}{v} d x d \tau \\
& \quad \leqq C_{3}\left(\left\|\varphi_{0}\right\|,\left\|\psi_{0}\right\|\right) .
\end{aligned}
$$

Proof of Lemma 4.1. Multiplying the first equation of (3.1) by $p(V)-p(V+\varphi)$ and the second one by $\psi$, summing them up and integrating it over $[0, t] \times R$, we have

$$
\begin{aligned}
& \int \frac{1}{2} \psi^{2}(t, x)+\Phi(v, V)(t, x) d x \\
& \quad+\int_{0}^{t} \int\left\{\mu \frac{\psi_{x}^{2}}{v}-\mu \frac{\varphi \psi_{x} V_{t}}{v V}+\left(p(V+\varphi)-p(V)-p^{\prime}(V) \varphi\right) V_{t}\right\} d x d \tau \\
& \quad \leqq \int_{2}^{1} \frac{1}{2} \psi_{0}^{2}(x)+\Phi\left(v_{0}(x), V(0, x)\right) d x+\int_{0}^{t}\left\|G(V)_{x}(\tau)\right\|\|\psi(\tau)\| d \tau
\end{aligned}
$$

If we put $p(V+\varphi)-p(V)-p^{\prime}(V) \varphi=f(v, V) \varphi^{2}$, then $f(v, V)>0$. Since

$$
\begin{aligned}
E & \equiv \mu \frac{\psi_{x}^{2}}{v}-\mu \frac{\varphi \psi_{x} V_{t}}{v V}+\left(p(V+\varphi)-p(V)-p^{\prime}(V) \varphi\right) V_{t} \\
& =\left(\sqrt{\mu} \frac{\psi_{x}}{\sqrt{v}}\right)^{2}-\frac{\sqrt{\mu V_{t}}}{V \sqrt{v f(v, V)}} \cdot \sqrt{\mu} \frac{\psi_{x}}{\sqrt{v}} \cdot \sqrt{f(v, V) V_{t}} \varphi+\left(\sqrt{f(v, V) V_{t}} \varphi\right)^{2},
\end{aligned}
$$

the discriminant $D$ is $\mu \frac{V_{t}}{V^{2} v f(v, V)}-4$, regarding $E$ as the quadratic equation. It holds $0<V_{t}<C \delta \varepsilon$ by Lemma 2.2 and $0 \leqq \frac{\mu}{V^{2} v f(v, V)} \leqq C<+\infty$ for $0<v<+\infty$. Hence we fix $\varepsilon>0$ so small as $D<0$. So, $E \geqq C^{-1}\left\{\left(p(V+\varphi)-p(V)-p^{\prime}(V) \varphi\right) V_{t}+\frac{\psi_{x}^{2}}{v}\right\}$ and $E \geqq C^{-1}\left|\frac{\varphi \psi_{x} V_{t}}{v V}\right|$. Thus (4.1) and (4.2) follows from the integrability of $\left\|G(V)_{x}(t)\right\|$ on $R_{+}$by virtue of Lemma 2.2. Q.E.D. 
Next, by the change of variable $\tilde{v}=v / V$ we reformulate (3.1) to another form:

$$
\left(\mu \frac{\tilde{v}_{x}}{\tilde{v}}-\psi\right)_{t}+\frac{\gamma \tilde{v}_{x}}{V^{\gamma} \tilde{v}^{\gamma+1}}+\frac{\gamma V_{x}}{V^{\gamma+1}}\left(\frac{1}{\tilde{v}^{\gamma}}-1\right)=g(V)_{x}-\mu\left(\frac{U_{x}}{V}\right)_{x}=-G(V)_{x} .
$$

If we multiply (4.6) by $\tilde{v}_{x} / \tilde{v}$ and integrate it over $R$, we have

$$
\left(\frac{\mu}{2} \int\left(\frac{\tilde{v}_{x}}{\tilde{v}}\right)^{2} d x\right)_{t}+\int\left\{-\psi_{t} \frac{\tilde{v}_{x}}{\tilde{v}}+\frac{\gamma \tilde{v}_{x}^{2}}{V^{\gamma} \tilde{v}^{\gamma+2}}+\frac{\gamma V_{x}}{V^{\gamma+1}}\left(\frac{1}{\tilde{v}^{\gamma}}-1\right) \frac{\tilde{v}_{x}}{\tilde{v}}\right\} d x \leqq\left\|G(V)_{x}\right\|\left\|\frac{\tilde{v}_{x}}{\tilde{v}}\right\| .
$$

Since

$$
\frac{\gamma V_{x}}{V^{\gamma+1}}\left(\frac{1}{\tilde{v}^{\gamma}}-1\right) \frac{\tilde{v}_{x}}{\tilde{v}} \leqq \frac{\gamma}{2} \frac{\tilde{v}_{x}^{2}}{V^{\gamma} \tilde{v}^{\gamma+2}}+\frac{\gamma}{2} \frac{V_{x}^{2}\left(1-\tilde{v}^{\gamma}\right)^{2}}{\tilde{v}^{\gamma} V^{\gamma+2}}
$$

and

$$
\int-\psi_{t} \frac{\tilde{v}_{x}}{\tilde{v}} d x=\int\left(-\psi \frac{\tilde{v}_{x}}{\tilde{v}}\right)_{t}-\psi_{x} \frac{\tilde{v}_{t}}{\tilde{v}} d x=\left(-\int \psi \frac{\tilde{v}_{x}}{\tilde{v}} d x\right)_{t}+\int\left(-\frac{\psi_{x}^{2}}{v}+\frac{\varphi \psi_{x} V_{t}}{v V}\right) d x,
$$

we have, by the integration of (4.7) over $[0, t]$,

$$
\begin{aligned}
& \int\left(\frac{\mu}{2}\left(\frac{\tilde{v}_{x}}{\tilde{v}}\right)^{2}(t)-\psi \frac{\tilde{v}_{x}}{\tilde{v}}(t)\right) d x+\int_{0}^{t} \int_{\frac{\gamma}{2}}^{\gamma} \frac{\tilde{v}_{x}^{2}}{V^{\gamma} \tilde{v}^{\gamma+2}} d x d \tau \\
& \leqq \\
& \int \frac{\mu}{2}\left(\frac{\tilde{v}_{0 x}}{\tilde{v}_{0}}\right)^{2}-\psi_{0} \frac{\tilde{v}_{0 x}}{\tilde{v}_{0}} d x+\int_{0}^{t}\left\|G(V)_{x}(\tau)\right\|\left\|\frac{\tilde{v}_{x}}{\tilde{v}}(\tau)\right\| d \tau \\
& \quad+\int_{0}^{t} \frac{\psi_{x}^{2}}{v}-\frac{\varphi \psi_{x} V_{t}}{v V}+\frac{\gamma}{2} \frac{V_{x}^{2}\left(1-\tilde{v}^{\gamma}\right)^{2}}{\tilde{v}^{\gamma} V^{\gamma+2}} d x d \tau,
\end{aligned}
$$

where $\tilde{v}_{0}(x)=v_{0}(x) / V(0, x)$. Due to Lemma 4.1 and Lemma 2.2, (4.8) yields

\section{Lemma 4.2.}

$\left\|\frac{\tilde{v}_{x}}{\tilde{v}}(t)\right\|^{2}+\int_{0}^{t} \int \frac{\tilde{v}_{x}^{2}}{V^{\gamma} \tilde{v}^{\gamma+2}} d x d \tau \leqq C_{4}\left(\left\|\varphi_{0}\right\|,\left\|\psi_{0}\right\|,\left\|v_{0 x}\right\|\right)+C \int_{0}^{t} \int \frac{V_{x}^{2}\left(1-\tilde{v}^{\gamma}\right)^{2}}{V^{\gamma+2} \tilde{v}^{\gamma}} d x d \tau$.

We now show the key lemma.

Lemma 4.3. Let $1 \leqq \gamma \leqq 2$. Then there exists a constant $C_{5}=C_{5}\left(\left\|\varphi_{0}\right\|,\left\|\psi_{0}\right\|,\left\|v_{0 x}\right\|\right)$ such that

$$
\begin{gathered}
\left\|\frac{\tilde{v}_{x}}{\tilde{v}}(t)\right\|^{2}+\int_{0}^{t} \int \frac{\tilde{v}_{x}^{2}}{V^{\gamma} \tilde{v}^{\gamma+2}} d x d \tau \leqq C_{5}, \\
C_{5}^{-1} \leqq v(t, x) \leqq C_{5} .
\end{gathered}
$$

Proof. We first prove the case when $\gamma=1$. Since $\left|V_{x}\right| \leqq C V_{t}$ and $p(V+\varphi)-p(V)-$ $p^{\prime}(V) \varphi=\frac{(1-\tilde{v})^{2}}{V \tilde{v}}$, the last term in (4.9) is finite by (4.1), which means (4.10). In order to show (4.11), we put

$$
\Psi(\tilde{v})=\int_{1}^{\tilde{v}} \tilde{\Phi}(\eta)^{1 / 2} \frac{d \eta}{\eta}
$$


following Kanel' [3]. Noting $\Psi(\tilde{v}(t, x)) \rightarrow 0$ as $x \rightarrow \pm \infty$, we have

$$
\begin{aligned}
|\Psi(\tilde{v}(t, x))| & =\left|\int_{-\infty}^{x} \frac{\partial}{\partial x} \Psi(\tilde{v}(t, x)) d x\right| \\
& \leqq \frac{1}{2}\left\|\frac{\tilde{v}_{x}}{\tilde{v}}(t)\right\|^{2}+\frac{1}{2} \int_{-\infty}^{\infty} \tilde{\Phi}(\tilde{v}(t, x)) d x .
\end{aligned}
$$

Since $\Psi(\tilde{v}) \rightarrow+\infty$ (respectively $-\infty$ ) as $\tilde{v} \rightarrow+\infty$ (respectively +0$),(4.11)$ is valid because of (4.10) and (4.4).

Next, we turn to the case $1<\gamma \leqq 2$, in which the procedure is more complicated. From (4.9) we lead to

Sublemma. For constants $C_{6}, C_{7}$ depending on $\left\|\varphi_{0}\right\|,\left\|\psi_{0}\right\|$ and $\left\|v_{0 x}\right\|$, it holds

$$
\sup _{\boldsymbol{R}} \tilde{v}(t, x) \leqq C_{6}+C_{7} \int_{0}^{t} \frac{1}{(1+\tau)^{2}} \cdot\left(\sup _{\boldsymbol{R}} \tilde{v}(\tau, x)\right)^{\gamma-1} d \tau .
$$

Proof. Let $\Omega_{1}(t)=\{x \in R ; \tilde{v}(t, x) \geqq 2\}, \Omega_{2}(t)=\left\{x \in R ; \frac{1}{2}<\tilde{v}(t, x)<2\right\}$ and $\Omega_{3}(t)=$ $\left\{x \in R ; \tilde{v}(t, x) \leqq \frac{1}{2}\right\}$, then we divide the integrand of the last term in (4.9):

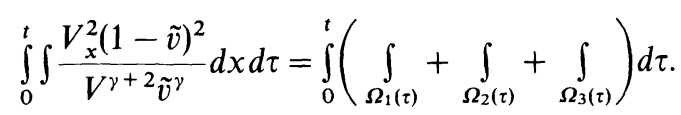

Since

$$
\left|\int_{0}^{t}\left(\int_{\Omega_{1}(\tau)}\right) d \tau\right| \leqq \int_{0}^{t}\left(\sup _{\boldsymbol{R}} \tilde{v}(\tau, x)\right)^{\gamma-1} \cdot\left\|V_{x}(\tau)\right\|_{L^{\infty}}^{2} \int_{\Omega_{1}(\tau)} \frac{\left(1-\tilde{v}^{\gamma}\right)^{2}}{\tilde{v}^{2 \gamma-1}} d x d \tau
$$

and

$$
\begin{aligned}
\int_{\Omega_{1}(\tau)} \frac{\left(1-\tilde{v}^{\gamma}\right)^{2}}{\tilde{v}^{2 \gamma-1}} d x & \leqq C \int_{\Omega_{1(\tau)}}\left(\tilde{v}-1+\frac{1}{\gamma-1}\left(\frac{1}{\tilde{v}^{\gamma-1}}-1\right)\right) d x \\
& \leqq \int_{R} C\left(v_{-}, v_{+}\right) V(\tau, x)^{1-\gamma} \tilde{\Phi}(\tilde{v}(\tau, x)) d x
\end{aligned}
$$

it is valid from (4.4) and Lemma 2.2

$$
\left|\int_{0}^{t}\left(\int_{\Omega_{1}(\tau)}\right) d \tau\right| \leqq C C_{3} \int_{0}^{t} \frac{1}{(1+\tau)^{2}} \cdot\left(\sup _{\boldsymbol{R}} \tilde{v}(\tau, x)\right)^{\gamma-1} d \tau .
$$

By the mean value theorem, it is easily seen

$$
\left|\int_{0}^{t}\left(\int_{\Omega_{2}(\tau)}\right) d \tau\right| \leqq C C_{3}
$$

When $0<\tilde{v} \leqq 1 / 2, \frac{\left(1-\tilde{v}^{\gamma}\right)^{2}}{\tilde{v}^{\gamma}} \leqq C\left(\tilde{v}^{\gamma}-1+\gamma(\tilde{v}-1)\right)$ and hence, due to (4.4),

$$
\left|\int_{0}^{t}\left(\int_{\Omega_{3}(\tau)}\right) d \tau\right| \leqq C C_{3}
$$


Combining (4.14)-(4.16), we have

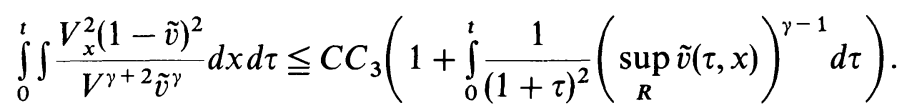

On the other hand, we also define $\Psi(\tilde{v})$ by (4.12) and note

$$
|\Psi(\tilde{v}(t, x))| \leqq\left\|\frac{\tilde{v}_{x}}{\tilde{v}}(t)\right\| \cdot\left(\int_{R} \tilde{\Phi}(\tilde{v}(t, x) d x)\right)^{1 / 2} \leqq C\left\|\frac{\tilde{v}_{x}}{\tilde{v}}(t)\right\|
$$

by (4.4). For a moment let $\tilde{v}(t, x) \geqq 2$, then

$$
\begin{aligned}
\Psi(\tilde{v}(t, x)) & =\int_{1}^{2} \tilde{\Phi}(\eta)^{1 / 2} \frac{d \eta}{\eta}+\int_{2}^{\tilde{v}(t, x)} \frac{\tilde{\Phi}(\eta)^{1 / 2}}{\eta} d \eta \\
& \geqq C+\int_{2}^{\tilde{v}(t, x)} C \eta^{-1 / 2} d \eta=2 C \tilde{v}(t, x)^{1 / 2}+C,
\end{aligned}
$$

so that

$$
\tilde{v}(t, x)^{1 / 2} \leqq C+C|\Psi(\tilde{v}(t, x))| .
$$

If we take $C \geqq \sqrt{2}$, then (4.19) holds also when $\tilde{v}(t, x)<2$. Applying (4.17)-(4.19) to (4.9) we obtain (4.13). Q.E.D.

Now, we can complete the proof of Lemma 4.3. If $\gamma-1 \leqq 1$, i.e. $\gamma \leqq 2$, then (4.13) shows $\sup \tilde{v}(t, x) \leqq C$ and so (4.10) due to. Lemma 4.2 and (4.17). Therefore, (4.11) follows from (4.18) in the same way as the case $\gamma=1$. Q.E.D.

The estimates of $\psi_{x}$ and $\psi_{x x}$ are obtained by the multiplication by $-\psi_{x x}$ to the second equation of (3.1) on the same line of [11]. We state the result only.

Lemma 4.4. There is a constant $C_{8}$ depending on $\left\|\varphi_{0}\right\|,\left\|v_{0 x}\right\|,\left\|\psi_{0}\right\|_{1}$ such that

$$
\left\|\psi_{x}(t)\right\|^{2}+\int_{0}^{t}\left\|\psi_{x x}(\tau)\right\|^{2} d \tau \leqq C_{8}
$$

Thus the proof of Proposition 3.2 is completed.

\section{References}

1. Hattori, Y., Nishihara, K.: A note on the stability of the rarefaction wave of the Burgers equation. Jpn. J. Appl. Math. 8, 85-96 (1991)

2. Il'in, A. M., Oleinik, O. A.: Asymptotic behavior of the solutions of the Cauchy problem for certain quasilinear equations for large time (Russian). Mat. Sb. 51, 191-216 (1960)

3. Kanel', Ya.: On a model system of equations of one-dimensional gas motion (Russian). Differencial'nya Uravnenija 4, 374-380 (1968)

4. Kawashima, S., Matsumura, A.: Asymptotic stability of travelling wave solutions of systems for one-dimensional gas motion. Commun. Math. Phys. 101, 97-127 (1985)

5. Kawashima, S., Matsumura, A., Nishihara, K.: Asymptotic behavior of solutions for the equations of a viscous heat-conductive gas. Proc. Jpn. Acad. 62, 249-252 (1986)

6. Lax, P. D.: Hyperbolic systems of conservation laws. II. Commun. Pure Appl. Math. 10, $537-566(1957)$ 
7. Liu, T.-P.: Nonlinear stability of shock waves for viscous conservation laws. Memoirs AMS 328, 1-108 (1985)

8. Matsumura, A., Nishida, T.: The initial value problem for the equations of motion of viscous and heat-conductive gases. J. Math. Kyoto Univ. 20, 67-104 (1980)

9. Matsumura, A., Nishida, T.: Periodic solutions in the half-space for a one-dimensional model of visco-elasticity (to appear)

10. Matsumura, A., Nishihara, K.: On the stability of traveling wave solutions of a onedimensional model system for compressible viscous gas. Jpn. J. Appl. Math. 2, 17-25(1985)

11. Matsumura, A., Nishihara, K.: Asymptotics toward the rarefaction waves of the solutions of a one-dimensional model system for compressible viscous gas. Jpn. J. Appl. Math. 3, 1-13 (1986)

12. Nishihara, K.: A note on the stability of travelling wave solutions of Burger's equation. Jpn. J. Appl. Math. 2, 27-35 (1985)

Communicated by S.-T. Yau 
